\title{
ABSTRACTS OF THE 5TH INTERNATIONAL CONFERENCE \\ ON ADVANCES IN \\ HEMATOLOGY AND ONCOLOGY \\ (ICAHO2017)
}

\author{
12-13 August, 2017, \\ Coeur d'Alene, Idaho, USA
}

Organized by

The Binaytara Foundation 
1

\section{PULMONARY EMBOLISM, A DIAGNOSIS TO REMEMBER IN SICKLE CELL DISEASE}

Julieta Munoz Mejia, Narayana Gandham

and Christine Junia

MacNeal Hospital, Berwyn, IL, U.S.A.

Background: Acute chest syndrome (ACS), a well-known medical emergency in sickle cell disease (SCD) presents as chest pain and dyspnea. SCD patients with pulmonary embolism (PE) present similarly, but PE is not the first diagnosis that physicians consider in this patient population. PE occurs in approximately $17 \%$ of SCD patients, while there is an increased risk in patients with sickle cell trait. Standard tools in approaching patients with $\mathrm{PE}$, such as the Wells score, are of limited use in SCD patients due to absence of other venous thromboembolism (VTE) risk factors. This results in a low calculated pre-test probability. D-Dimer assay, commonly used to exclude PE, is also inherently elevated in SCD. Methods: A 23-year-old female patient with history of sickle cell disease presented with lumbar pain, dyspnea, and palpitations. Physical exam revealed tachycardia and tachypnea. Diagnostic work-up revealed white blood cell (WBC) count $9.7 \times 10^{3}$ cells $/ \mu \mathrm{l}$, hemoglobin $(\mathrm{Hgb}) 8.9 \mathrm{~g} / \mathrm{dl}$, and reticulocytes of $4.8 \%$. Chest X-ray was normal. The patient was treated with intravenous (IV) fluids and pain management. On hospital day 2, the patient became febrile at $39.4^{\circ} \mathrm{C}$, had persistent tachycardia, and was hypoxic to $91 \%$ on room air. Repeat infectious work-up and chest X-ray were unremarkable. The patient was empirically treated with IV ceftriaxone. Wells criteria showed low probability of PE at $1.5 \%$. Arterial blood gas (ABG) analysis revealed an elevated $\mathrm{P}(\mathrm{A}-\mathrm{a}) \mathrm{O}_{2}$ Gradient and chest computed tomography (CT) revealed pulmonary embolism. Finally, Eliquis was administered and patient improved with anticoagulation. Results: Limited data or guidelines are available in regards to PE diagnosis in patients with SCD resulting in delay of diagnosis and life-saving anticoagulation treatment. Conclusion: Physicians should maintain a high index of suspicion for PE in SCD patients that present with acute respiratory symptoms. Tests of higher specificity, such as chest CT scan, should be considered first line for diagnosis, especially since current prediction tools underestimate the pre-test probability of PE in the specific patient group.

\section{2}

ANDROGEN-SECRETING ADRENAL ADENOMA: CASE REPORT AND REVIEW OF LITERATURE

Subash Ghimire, Manidhar Lekkala, Amir Bista, Swapna Talluri, Zachary Wolfe and Kerri Kissell

Guthrie Robert Packer Hospital, Sayre, PA, U.S.A.
Background: Pure androgen-secreting adrenal adenoma (PASAA) is an extremely rare condition. We present an interesting case of a female patient presented with androgenic symptoms and resolution of the symptoms after removal of the adrenal adenoma. Case Report: A 43-year-old female was referred from the primary care clinic after incidental finding of left adrenal mass during work-up for nephrolithiasis. Clinical history included rapidly progressing facial hair growth, acne, and voice deepening. On physical examination, the patient was hemodynamically stable and body mass index was $31 \mathrm{~kg} / \mathrm{m}^{2}$. Abdominal computed tomography (CT) scan showed left adrenal mass measuring $7.6 \mathrm{~cm} \times 7 \mathrm{~cm} \times 8 \mathrm{~cm}$ (Figure 1). Lab tests showed elevated testosterone (TT) level of $271 \mathrm{ng} / \mathrm{dl}$ (normal levels 8-48) and free-TT level of $7.3 \mathrm{pg} / \mathrm{ml}$ (normal levels 0.0-2.2). The patient underwent open surgical exploration and removal of the adrenal mass. Histopathology of the resected specimen showed tan-yellow, ovid soft tissue mass with focal hemorrhages, necrosis and cystic degeneration surrounded by a rim of normal appearing, golden yellow cortex. Microscopic examination was performed at the National Institute of Health (NIH). Clear cells with diffuse architecture were seen with mild atypia, below nuclear grade 3 were observed. A pathologic diagnosis of adrenal cortical adenoma was made. Follow-up examination revealed resolution of hyperandrogenic symptoms and normalization of serum androgen levels. Discussion: Less than fifty cases of PASAA have been described so far (1). Most of the adrenal tumors are incidentally found on imaging studies like CT scan, magnetic resonance imaging and ultrasonogram (2). Occasionally, PASAA is diagnosed during evaluation of hyperandrogenic symptoms. Hyperandrogenic symptoms in females include excessive facial hair growth, male pattern baldness and virilization symptoms (deepening of voice, clitoromegaly, menstrual irregularities, change in voice) (2). The standard of treatment for adrenal tumors, including PASAA is

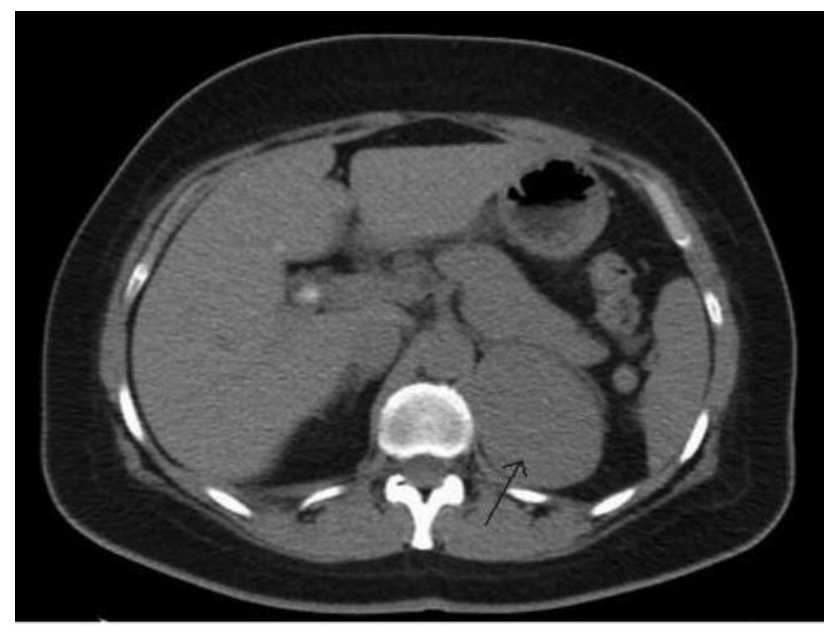

Figure 1. Computerized tomography (CT) scan showing left adrenal mass. 
surgical resection (3). Adjuvant radiotherapy to the tumor bed is recommended in incomplete resections. Adjuvant chemotherapy with systemic mitotane is recommended for patients with incomplete surgical resection and high-risk features (Ki67 gene expression >10\%) (3). Patients with inoperable tumor, rapid disease progression, and high volume of tumor should be treated with combination chemotherapy including etoposide, doxorubicin, cisplatin and mitotane (EDPMitotane) (3). Patients should be followed up at regular intervals with monitoring of hormone level and imaging studies. Conclusion: Radiology is the mainstay of PASAA diagnosis and surgical resection is the standard for treatment. Adjuvant radiotherapy and chemotherapy are indicated only in incomplete resection or rapid disease progression. Regular follow-up after treatment with hormone level monitoring is recommended.

1 Moreno S, Montoya G, Armstrong J, Leteurtre E, Aubert S, Vantyghem MC, Dewailly D, Wemeau JL and Proye C: Profile and outcome of pure androgen-secreting adrenal tumors in women: Experience of 21 cases. Surgery 136(6): 1192-1198, 2004.

2 Tong A, Jiang J, Wang F, Li C, Zhang Y and Wu X: Pure androgen-producing adrenal tumor: Clinical features and pathogenesis. Endocr Pract 23(4): 399-407, 2017.

3 Berruti A, Baudin E, Gelderblom H, Haak HR, Porpiglia F, Fassnacht M, Pentheroudakis G and ESMO Guidelines Working Group: Adrenal cancer: ESMO Clinical Practice Guidelines for diagnosis, treatment and follow-up $\dagger$. Ann Oncol 23(Suppl 7): vii131-vii138, 2012.

3

\section{ANTI-MALARIAL DRUGS ARTESUNATE AND CHLOROQUINE INDUCE CELL DEATH BY DIFFERENT MECHANISMS IN BILE DUCT CANCER CELL LINES}

Diwakar Guragain ${ }^{1,2,3}$, Norie Araki ${ }^{3}$, Atit Silsinivanit $^{1,2,3}$, Wunchana Seubwai ${ }^{2,4}$ and Ubon Cha` on ${ }^{1}$

${ }^{1}$ Department of Biochemistry, Faculty of Medicine, Khon Kaen University, Khon Kaen, Thailand; ${ }^{2}$ Liver Fluke and Cholangiocarcinoma Research Center, Khon Kaen University, Khon Kaen, Thailand; ${ }^{3}$ Department of Tumor Genetics and Biology, Graduate School of Medical Sciences, Kumamoto University, Kumamoto, Japan; ${ }^{4}$ Department of Forensic Medicine, Faculty of Medicine, Khon Kaen University, Khon Kaen, Thailand

Background: Pathogenesis of cholangiocarcinoma (CCA), a malignancy of bile duct epithelial cells, is multifactorial. CCA in the Northeast Thailand is associated with chronic inflammation due to liver fluke (Opisthorchis viverrini, OV) infection. Since chemotherapy for OV-associated CCA is not quite successful, we revisited the efficacy of antimalarial drugs, artesunate (ART) and chloroquine (CQ), against CCA. ART and CQ are the most explored among anti-parasitic drugs for their anti-cancer effects and have shown potential of inhibiting cell growth and inducing cell death in various cancer cells. ART and CQ, both induce cell death of malaria parasite by a similar mechanism depending upon formation of reactive oxygen species (ROS). Thus, it is of great interest to study whether the cell death mechanisms of bile duct cancer cells after treatment with ART and CQ are similar or different. Methods: The effects of ART and CQ were studied on bile duct cancer cell lines M214L0 and M214L5. Cell proliferation after treatment with ART and CQ was studied by WST assay. The phenotypic cell death pattern was studied using time-lapse analysis. Results: Both ART and CQ inhibited proliferation of bile duct cancer cell lines M214L0 and M214L5 in a dose- and time-dependent manner. Regarding the phenotypic pattern of cell death, ART induced necrosis, whereas CQ induced apoptosis, against both bile duct cancer cell lines. Conclusion: ART and CQ induce cell death in bile duct cancer cell lines via different mechanisms. However, the different cell death patterns induced by ART and $\mathrm{CQ}$ need to be further explored at the molecular phenotypic level.

\section{4}

\section{UNUSUAL RELAPSE OF CHRONIC LYMPHOCYTIC LEUKEMIA AFTER REMISSION}

\author{
Wajeeha Rizvi ${ }^{1}$ and Quoc Van Truong ${ }^{1,2}$ \\ ${ }^{1}$ University of Kansas School of Medicine, \\ Wichita, KS, U.S.A.; \\ ${ }^{2}$ Cancer Center of Kansas, Manhattan, KS, U.S.A.
}

Background: Chronic Lymphocytic Leukemia (CLL) is the most prevalent leukemia with over 20,000 estimated cases in 2017. Leukemic involvement of the nervous system causing neurological symptoms is reported in only about $1 \%$ of CLL patients. Unfortunately, there is no current standard therapy for treatment of CLL leptomeningeal disease. In this case, we discuss an unusual presentation of CLL leptomeningeal disease misdiagnosed as chronic rebound headache. Case Case Report: A 61-year- old female was diagnosed with Rai stage I CLL in 2002. When presented with peripheral blood lymphocytosis and subsequent flow cytometry revealed a mature B cell population consistent with CLL. The patient was monitored clinically as there were no indications for therapy. In 2006, the patient developed B symptoms along with hemolytic anemia refractory to steroids and was initiated on chemotherapy with fludarabine, cyclophosphamide, and rituximab (FCR), showing complete response after six cycles. The patient was in her usual state 
of health until 2016, when complained of chronic headaches. Tylenol and ibuprofen were administered regularly and the patient was diagnosed with rebound headaches by neurology. These symptoms progressed and the patient developed encephalopathy requiring inpatient admission. Magnetic Resonance Imaging (MRI) revealed abnormal enhancement in the cerebellar peduncles and dentate nuclei symmetrically and lumbar puncture performed revealed evidence of CLL consistent with leptomeningeal disease. Therapy was started with oral ibrutinib at $560 \mathrm{mg}$ daily for better central nervous system penetration. After three months of therapy, the patient had complete resolution of symptoms and MRI abnormalities. Conclusion: Leptomeningeal disease is a rare complication of CLL that clinicians should be aware of and ibrutinib can be an effective, tolerable therapy for this debilitating disease.

\section{5}

\section{RECURRENT PLEURAL AND PERICARDIAL EFFUSIONS IN A PATIENT WITH A RIGHT ATRIAL ANGIOSARCOMA: A CASE REPORT}

\author{
Kristen Carroll ${ }^{1}$, Chad Kloefkorn ${ }^{2}$ and Jigna Zatakia ${ }^{3}$ \\ ${ }^{1}$ School of Medicine, Baylor College of Medicine, \\ Houston, TX, U.S.A.; \\ ${ }^{2}$ Department of Internal Medicine, Baylor College of \\ Medicine, Houston, TX, U.S.A.; \\ ${ }^{3}$ Division of Pulmonary Critical Care and Sleep Medicine, \\ Baylor College of Medicine, Houston, TX, U.S.A.
}

Background: Angiosarcomas are rare endothelial vascular malignancies composed of anaplastic epithelial cells. These aggressive tumors have wide metastatic potential via both hematogenous and lymphatic spread. They tend to occur between the third and fifth decades of life, more frequently in men. Although most commonly originating in soft tissue and skin, angiosarcomas can develop from the pericardium, liver, spleen or even breast. Angiosarcomas represent the most common type of primary cardiac malignancy but overall are extremely rare and often fatal. Methods: We present a case of a 36-year-old previously healthy male who initially presented with bilateral lower extremity edema and was found to have large recurrent pericardial effusions. The pericardial fluid was predominately bloody with scant cellularity, cultures were negative, and cytology did not reveal malignant cells. Pericardial biopsy showed nonspecific chronic inflammation. His effusion was thought to be due to post-infectious immune-mediated pericarditis, for which he was given pulse dose steroids and discharged on colchicine. Four months later he presented with subacute cough and was found to have a large left-sided blood exudative pleural effusion, again with negative cultures and cytology, eventually requiring pleurodesis. An extensive rheumatologic work-up, tumor markers, pleural and lung biopsies were non-diagnostic. MRI revealed a right atrial irregularity thought to be a clot and further imaging showed multiple small hypodense liver lesions. The patient was discharged on anticoagulation, but was lost to follow-up, only to return two months later with abdominal pain and hyperbilirubinemia. Imaging revealed multiple liver masses with extravasation suggesting hepatic metastases for which he underwent laparoscopy with hemoperitoneum evacuation. Surgical pathology was consistent with angiosarcoma involving the pericardium, right middle lung and liver, most likely of primary right atrial or pericardial origin. Results: Although rare, the majority of cardiac angiosarcomas originates in the right atrium and can invade local structures, including the pericardium. Patients typically present late after significant tumor progression because the initial symptoms are nonspecific and are frequently misdiagnosed as acute pericarditis. Conclusion: The differential diagnosis for recurrent exudative pleural and pericardial effusions is short and includes infection, pulmonary embolism, collagen vascular disease and malignancy, notably cardiac angiosarcomas. Patients with cardiac angiosarcomas exhibit rapid progression of symptoms and advanced clinical deterioration. Prognosis for angiosarcoma is very poor: mean survival is estimated at 6-14 month with a 5-year survival rate of only $14 \%$. Cardiac magnetic resonance imaging is better than computed tomography in identifying an atrial abnormality, but still cannot reliably differentiate a thrombus from a tumor, thus diagnosis frequently requires surgical pathology. The absence of malignant cells on fluid cytology, normal pericardial/pleural biopsies, and negative tumor markers cannot rule out an angiosarcoma.

\section{6}

\section{LEPTOMENINGEAL CARCINOMATOSIS ASSOCIATED WITH GALL BLADDER CARCINOMA: A CASE REPORT AND REVIEW OF THE LITERATURE}

Talal Khan, Saleha Rizwan, Manik Veer, Mohammad Bilal and Scott Long

Allegheny Health Network, Pittsburgh, PA, U.S.A.

Background: Gallbladder carcinoma (GBC) ranks sixth among gastrointestinal cancers with a poor prognosis beyond initial stages. The most common site of metastasis for GBC is the liver, while involvement of the leptomeninges is extremely rare. Leptomeningeal carcinomatosis (LMC) in GBC portends a poor prognosis with anecdotal evidence for therapy and outcomes. We present a case of stage IV gallbladder carcinoma (GBC) with leptomeningeal involvement and review all the previously described cases of this entity. Case Report: A 67-year-old Caucasian female with history of gallbladder carcinoma 
diagnosed 16 months ago, presented to the hospital with two days of dysarthria, confusion, lethargy and frequent headaches of four weeks duration. Patient had received chemotherapy with four cycles of gemcitabine and cisplatin, most recently three months ago. The patient was disoriented and unable to follow commands. Neurological exam was difficult due to the inability of the patient to follow commands. A computed tomography (CT) scan of the head without contrast was normal. MRI of the brain with contrast revealed nodular leptomeningeal enhancement which was most pronounced in the cerebellum, quadrigeminal plate cistern, and bilateral occipital lobes. A lumbar puncture showed high protein, low glucose, and high WBCs, though cultures remained negative. Cytology of CSF confirmed malignant cells consistent with adenocarcinoma. Cytokeratin stain and Leukocyte Antigen Stain were positive confirming a diagnosis of LMC secondary to GBC. Palliative whole-brain radiation therapy (WBRT) was attempted, but no improvement was noted in patient's neurological function. Decision was made to stop further therapy and patient was transitioned to hospice care. Discussion: LMC secondary to solid tumors is rare and seen in 5-8\% of breast cancers, $6-18 \%$ of malignant melanomas and $9-25 \%$ of lung cancers. Thus far, only 10 cases of leptomeningeal spread of GBC have been reported. These reports document various time intervals for the progression from GBC to $\mathrm{LMC}$, between 3-72 months $(\mathrm{n}=4)$, with a mean survival of 53 days $(n=6)$. Symptoms have been variable and range from painless visual loss to lower motor neuron lesions of the limbs. Histological analysis has included diffuse, nodular, and mixed types of LMC $(n=6)$. Although therapeutic options for LMC include intracranial implantation of reservoirs for intrathecal chemotherapy administration, ventriculoperitoneal shunts, radiation therapy (RT) and systemic chemotherapy, there is no standardization of therapy for GBCassociated LMC. Conclusion: Internists need to be cognizant to cancer associated complications such as LMC which may masquerade as meningitis. Rapid clinical decompensation and a short mean survival rate necessitate early diagnosis. Early initiation of palliative chemotherapy and radiation therapy with a palliative consult seem to be necessary when treating GBC associated LMC. Furthermore, early diagnosis is imperative for appropriate family counselling.

\section{7}

\section{HEPARIN-LIKE ANTICOAGULANT IN MULTIPLE MYELOMA, RESPONSIVE TO BORTEZOMIB TREATMENT}

Talal Khan, Saleha Rizwan, Manik Veer, Santosh Sadashiv and Robert Kaplan

Allegheny Health Network, Pittsburgh, PA, U.S.A.

Background: Major bleeding as a complication of multiple myeloma (MM) is uncommon. Various etiologies like the heparin-like anticoagulant have been described to explain the coagulopathies. Herein, we describe our experience in the management of such condition and the role of chemotherapy, highlighting the importance of considering preoperative coagulation testing to avoid potentially fatal complications as postoperative hemorrhage. Case Report: A 73-year-old Caucasian female presented with hip fracture and was eventually diagnosed with stage III MM, according to the international staging system (ISS). The patient underwent open reduction and internal fixation of the femur and post-operative course was complicated with surgical site bleeding requiring multiple packed red blood cells (PRBC's) and platelet transfusions. Further testing revealed a prolonged activated partial thromboplastin time (aPTT) without correction on mixing study, normal fibrinogen, prolonged thrombin time (TT), without full correction on hepabsorb and a prolonged reptilase time (RT). These findings were suggestive of heparin-like anticoagulant. Multiple therapies including fresh-frozen plasma (FFP) administration, DDAVP, hemodialysis (HD), total plasma exchange and protamine sulfate did not result in clinical improvement. The patient received five cycles of bortezomib and upon completion of chemotherapy, major bleeding from the post-operative site stopped. The patient made a successful recovery and was able to be discharged home. Discussion: Multiple etiologies of coagulopathy should be considered when evaluating hemorrhage in patients with MM. Defects may include dysfibrinogenemia, platelet dysfunction, tissue paraprotein infiltration, acquired Von Willebrand's and heparin-like anti- coagulant. These entities must be a part of differential diagnosis during evaluation of hemorrhage in patients with MM. Heparinlike anticoagulant syndrome is rare with no well described management guidelines and treatment is rendered based on case reports or individual experiences. Few case reports and in vitro studies have suggested benefits with use of therapeutic plasma exchange (TPE), hemodialysis and protamine sulfate. Based on our experience we would like to emphasize that the benefits from these interventions are marginal at best and are derived from aggressive treatment of the underlying MM with novel proteasome inhibitor agents. Bortezomib-based regimens should be strongly considered and immediately administered in such patients. Hemorrhage should not delay or preclude the use of bortezomib-based therapy. Conclusion: It is imperative for an internist to consider intrinsic coagulopathy as part of pre-operative evaluation in MM patients. A preoperative coagulation panel in these patients could consist of bleeding time, PT/INR, aPTT, TT and RT in addition to mixing studies. Timely diagnosis of underlying coagulopathy could prevent major complications like postoperative hemorrhage which, in many cases, could turn out to be fatal. 
8

\section{METASTASIS TO PROSTATIC FOSSA FROM ADENOCARCINOMA OF THE DUODENUM - A RARE CASE REPORT}

\author{
Hafiz Khan, Ahsan Wahab, Siddique Chaudhary, \\ Mahin Khan and Susan Smith
}

McLaren-Flint/Michigan State University, Flint, MI, U.S.A.

Background: Metastases to the prostatic fossa (PF) from primary duodenal adenocarcinoma (DA) is a rare entity. As per our knowledge, there has been no previously reported case. We describe a patient with DA metastatic to the PF involving the urinary bladder. Methods: In April, 2014, a 76year-old African-American male with a history of prostate cancer, status post-surgery and radiotherapy presented with nausea, vomiting, abdominal pain and was diagnosed with small bowel obstruction. Computed tomography (CT) abdomen showed $5 \times 1 \mathrm{~cm}$ obstructing lower duodenal mass with mesenteric lymphadenopathy. Carcinoembryonic antigen (CEA) and cancer antigen (CA) $19-9$ were $7.7 \mathrm{ng} / \mathrm{ml}$ and $431.6 \mathrm{U} / \mathrm{ml}$, respectively. Exploratory laparotomy showed an obstructing mass adherent to the jejunum and left colon, thus en bloc duodeno-jejunal resection and left hemicolectomy with primary anastomoses were performed. Histopathology of the specimen showed well-differentiated adenocarcinoma. Adjuvant chemotherapy FOLFOX (folinic acid, fluorouracil, and oxaliplatin) was administered based on the Stage III-B the disease. Positive emission tomography (PET)-CT and tumor markers were negative in May 2014. Patient was followed-up with CEA, CA 19-9 and PET-CT. In May 2016, CEA and CA 19-9 were elevated while PETCT showed mildly increased metabolism in the PF without bladder involvement. Repeat PET-CT in August 2016 showed increased metabolism with involvement of the urinary bladder neck, while PSA was normal. The patient underwent cystoscopy with tumor biopsy of the bladder neck. Histopathology showed invasive moderately differentiated adenocarcimona. Immunostaining was positive for CK20 and CDX2 while negative for prostate specific antigen (PSA) and prostatic specific acid phosphatase (PSAP), which was consistent with the origin of the tumor from the DA. Patient opted for non-surgical treatment and was started on chemotherapy with FOLFIRI (folinic acid, fluorouracil and irinotecan) and bevacizumab. Results: DA is an uncommon neoplasm with a prevalence of $0.35 \%$ and $30-40 \%$ among gastrointestinal and small intestinal tumors, respectively. Its vague symptoms can delay the diagnosis. Many (40-65\%) of DA have regional nodal metastasis at diagnosis. The exact mechanism of distant metastasis is unclear. However, it metastasizes, most commonly to the liver, but also to the lungs and rarely to large vessels like the superior vena cava. PET-CT imaging is useful for detecting both primary DA and metastasis. DA is usually treated with pancreaticoduodenectomy with lymph node dissection, if localized, while adjuvant chemotherapy is indicated in metastasis. Patients with stage III and IV disease have decreased survival rate compared with those with stage $0, \mathrm{I}$, and II disease. Conclusion: Duodenal adenocarcinoma metastasis can present with atypical symptoms. To the best of our knowledge, this case is the first DA with PF metastasis to be reported. In our patient the clinical course, radiological features, and histopathological characteristics were consistent with his previously treated DA.

\section{9}

\section{BING-NEEL SYNDROME - A RARE PRESENTATION OF LYMPHOPLASMACYTIC LYMPHOMA}

\author{
Jyothi Margapuri ${ }^{1}$, Neeraj Saini ${ }^{2}$ and Venu Bathini ${ }^{2}$ \\ ${ }^{1}$ Icahn School of Medicine at Mount \\ Sinai, New York, NY, U.S.A.; \\ ${ }^{2}$ University of Massachusetts, Worcester, MA, U.S.A
}

Background: Bing-Neel syndrome (BNS) is an extremely rare complication of Waldenstrom's macroglobulinemia (WM) which is associated with direct central nervous system (CNS) infiltration by malignant lymphoplasmacytoid and plasma cells with or without cerebrospinal fluid (CSF) hyperglobulinemia. Case Report: A 69-year- old man with history of hypertension, benign schwannoma of the trigeminal nerve, status post radiotherapy, with no recurrence, lumbago with vertebral compression fracture, was admitted with chronic progressive leg weakness with gait abnormalities. The patient also complained of unintentional weight loss of 50 pounds in one year and denied any sensory loss, fecal and urine incontinence, fever, chills, blurry vision and rest all review of systems were negative. Physical examination was remarkable for nystagmus. Initial investigations revealed chronic normocytic anemia, elevated blood urea nitrogen and serum creatinine. Iron panel showed elevated ferritin, however, iron, total iron binding capacity, transferrin and lactate dehydrogenase were decreased. Serum protein electrophoresis with immunofixation revealed IgM kappa monoclonal gammopathy $(0.9 \mathrm{~g} / \mathrm{dl})$. Light chain ratio showed elevated kappa light chain with normal lambda light chain. Flow cytometry of CSF fluid revealed no clonal neoplastic cells, however, cytology showed small lymphocytes and monocytes and rare plasma cells that raised concern for possible CNS involvement. Positivity for MYD88 mutation confirmed the presence of lymphoplasmacytic lymphoma involving the CNS. Imaging, including chest, abdomen and pelvis computed tomography (CT), showed retroperitoneal lymphadenopathy suspicious for malignancy. Magnetic resonance imaging of 
lumbar/ thoracic spine revealed multifocal enhancing nodular thickening of cauda equina, concerns for leptomeningeal involvement, focal lesion in T11-L1 seen without any cord compression or stenosis. Bone marrow biopsy showed lymphoplasmacytic lymphoma with presence of a lymphoplasmacytoid and plasma cells infiltration $~ 50-$ $55 \%$ and monoclonal $\operatorname{IgM}(\mathrm{k})$. Results: The patient was started on methotrexate with oral Leucovorin and subsequently received chemotherapy Velcade/Dexamethasone/ Rituxan cycle and intrathecal cytarabine for CNS prophylaxis. IgM levels decreased after three cycles of therapy. Discussion: Bing-Neel syndrome is a rare heterogeneous entity with involvement of LPL in the CNS and can present as either in a diffuse or tumoral form involving the CNS. The distinction is mainly based on imaging data. Pathophysiology remains unexplained, but the role of hyperviscosity could explain the disruption of the blood-brain barrier with subsequent infiltration of LPL cells. Neurological complications are dominated by IgM-related neuropathies such as demyelinating peripheral neuropathy with IgM antibody activity against myelin-associated glycoprotein, along with rare direct CNS involvement by malignant lymphoid cells, like in the present case. Treatment includes various chemotherapy regimens such as rituximab/bendamustine +/- bortezomib that are used to treat LPL; however, CNS penetration remains poor. Systemic or intrathecal methotrexate is the mainstay of treatment for the CNS involvement. Recently, ibrutinib and lenalidomide have proved effective in the treatment of LPL with good CNS penetration. Conclusion: BNS is a probably under-recognized clinico-pathological entity and its diagnosis remains challenging with poor prognosis, especially when the patient is presented without a known history of WM. Clinicians should have high suspicion for this rare diagnosis.

\section{0 \\ WHEN LIGHTENING STRIKES TWICE: A CASE OF HIGH-GRADE MENINGIOMA WITH RAPID RECURRENCE IN A PREVIOUSLY BENIGN TUMOR}

Shahzeem Bhayani, Adnan Asif Parvez Ghias and Sudershan Garg

St. Elizabeth Youngstown Hospital, Youngstown, OH, U.S.A

Background: Meningiomas are the most common primary brain tumor. Historically, these tumors are considered mostly benign and gross total resection (GTR) remains the standard of care. However, with advent of newer imaging and pathological sub-classification there has been an increasing interest into managing higher grades of these tumors more aggressively. Due to the rare nature of newly diagnosed high grade meningioma which accounts for 300 cases in the United States, no prospective clinical trial has been conducted so far to determine whether adjuvant radio or chemotherapy could be beneficial $(1,2)$. Most data in favor of adjuvant RT are based on retrospective trials. We present a case of a patient that was initially treated with GTR though later recurred with a higher grade of the disease. Case Report: A 72-year-old female with past medical history of hypertension, hyperlipidemia, myotonic dystrophy, recurrent meningiomas, presented to the emergency department after a sudden worsening of rightsided weakness. Patient was recently discharged from acute rehabilitation following excision of a meningioma. The patient had a history of recurrent left frontoparietal meningiomas and had undergone two craniotomies with excisions. The first craniotomy with excision of WHO grade II meningioma was one year and five months ago. The patient had a second surgery done about two months ago revealing an anaplastic grade III meningioma. On examination, the patient had right sided hemiparesis, while the rest of the examination was unremarkable. The patient then underwent a CT head scan showing intracranial hemorrhage and left parietal mass. MRI demonstrated a large heterogeneous left parietal mass adjacent to recent craniotomy measuring $5 \times 4 \times 5.9 \mathrm{~cm}$ with surrounding hemorrhage and edema. There was also $2 \times 1.2 \times 1.2 \mathrm{~cm}$ mass anterior to left frontal lobe. This was followed by a left parietal craniotomy to excise the recurrent tumor and evacuation of hemorrhage. The pathology report demonstrated anaplastic meningioma - WHO grade III. The patient was then admitted in acute rehabilitation with oncology recommendation to initiate radiation therapy. Discussion: Meningiomas are mostly benign and make up for $37 \%$ of the primary brain tumors (3). However, there are rare cases of meningiomas, approximately $1 \%$ of all primary brain tumors, which, based on the WHO classification, can be atypical and anaplastic. Surgical resection is considered curative for all the types of meningiomas (4, 5). However, along with surgical resection, radiotherapy is essential for high-grade meningiomas, since the latter are aggressive in nature, and anaplastic meningiomas have the worst prognosis of the three grades. We present a case of malignant meningioma with a rapid recurrence in two months. Since malignant meningiomas are uncommon and rapid recurrence is even rarer, it is important to identify these tumors on the histological basis in order to provide appropriate care. Moreover, radiotherapy should be prioritized in these patients with a very close follow-up planned for monitoring. Conclusion: As expected, the present case demonstrated that malignant meningiomas have higher recurrence rates and poorer prognosis, compared to their benign counterparts. Combination of both surgical excision 
and radiotherapy in our patient can potentially help with longer recurrence-free period. Clinicians should be aware of radiotherapy indications in high risk patients and use histological grading with Ki-67 antigen expression to further guide clinical judgment due to its prognostic value in outcome. The appropriate coordinated care will reduce morbidity in such patients and prolong survival with achieving longer recurrence-free periods.

1 Rogers L, Barani I, Chamberlain M, Kaley TJ, McDermott M, Raizer J, Schiff D, Weber DC, Wen PY and Vogelbaum MA: Meningiomas: knowledge base, treatment outcomes, and uncertainties: A RANO Review. J Neurosurg 122(1): 4-23, 2015.

2 Wang J, Wang L, Luo B, Chen Z, Xiong Z, Fang M and Li $\mathrm{J}$ : Recurrent meningioma with malignant transformation: a case report and literature review. Int J Clin Exp Med 8(9): 16845-16849, 2015

3 Cancer.net. Meningioma: Statistics. [online] Available from: http://www.cancer.net/cancer-types/meningioma/ statistics [Accessed 6 Aug. 2017].

4 Walcott BP, Nahed BV, Brastianos PK and Loeffler JS: Radiation treatment for WHO grade II and III meningiomas. Front Oncol 3: 227, 2013.

5 Wang YC, Chuang CC, Wei KC, Chang CN, Lee ST, Wu CT, Hsu YH, Lin TK, Hsu PW, Huang YC, Tseng CK, Wang CC, Chen YL and Chen PY: Long term surgical outcome and prognostic factors of atypical and malignant meningiomas. Sci Rep 6: 35743, 2016.

\section{1}

\section{ADVANCED LOBULAR BREAST CARCINOMA WITH METASTASIS TO THE ORBIT: A CASE REPORT}

Ahsan Wahab, Siddique Chaudhary, Mahin Khan, Hafiz Khan and Susan Smith

McLaren Regional Medical Center, Flint, MI, U.S.A.

Background: Metastatic breast cancer (MBC) is the most common cause of malignancy-related mortality in females. However, orbital metastases with $\mathrm{BC}$ are extremely rare. Methods: A 75-year-old Caucasian female with recurrent metastatic infiltrative lobular BC (ILBC) had significant disease progression despite multiple therapies over many years. The patient had diffused osseous and subcutaneous metastasis and was being treated with palliative endocrine therapy. Over two months, a left orbital soft tissue lesion was developed causing proptosis. Magnetic resonance imaging (MRI) brain revealed calvarial as well as leptomeningeal metastatic disease, while MRI of the orbits showed enhancement of the lateral and superior rectus muscles, soft tissue, and lateral wall of the left orbit. In view of the grave prognosis, hospice care was opted and patient died in a few weeks. Results: Of all metastatic disease to the orbit, $\mathrm{BC}$ accounts for $50 \%$, though only $6 \%$ of MBC involve the orbits. While ILBC comprises 10-15\% of all BC, estrogen positive ILBC constitutes almost all BC metastasizing to the orbit suggesting some unique tumor behavior of ILBC. Leptomeningeal carcinomatosis can concomitantly exist, as in our patient. Symptoms include diplopia, dysmotility, pain and/or proptosis. Presentation can mimic other inflammatory, infiltrative or autoimmune disorders, but may represent a vast array of neoplastic lesions including metastatic disease. Imaging, like computed tomography (CT) and MRI, and biopsy are key to diagnosis. Treatment modalities include radiation, chemotherapy, hormonal therapy, surgery or a combination but should be tailored to individual cases depending on the patient's goals and overall prognosis. Conclusion: Although rare, orbital metastasis should be considered in all the patients with advanced BC presenting with orbital complaints, particularly those who show progression despite being treated with multiple agents. It is also imperative to understand the spectrum of orbital involvement in metastatic disease and investigate accordingly. Even if discovered earlier, the diagnosis of orbital metastases signifies a poor prognosis due to the concomitant presence of leptomeningeal carcinomatosis and systemic disease.

\section{2}

\section{RETROSPECTIVE ANALYSIS OF INDICATIONS OF IVC FILTER PLACEMENT AT OUR INSTITUTION}

Saleha Rizwan, Talal Khan, Rama Bhagavatula, Daniel Lee and Swapna Goday

Allegheny Health Network, Pittsburgh, PA, U.S.A.

Background: Placement of inferior vena cava (IVC) filters where other methods of thrombo-prophylaxis are presumed inadequate, technically challenging, or place patients at high risk for bleeding, has rapidly increased. As of 2012, the number of IVC filters placed in the United States was 25 times more than Germany, France, UK, Italy and Spain combined. IVC filters remain the only option for patients with acute venous thromboembolism (VTE) who have an absolute contraindication to anticoagulation. In such patients, IVC filters should be removed and anticoagulation resumed once the contraindication has passed. Despite lack of evidence, the rate of IVC filter placement for prophylaxis has increased. Evidence suggests their use is associated with clinically significant adverse events and increased health care costs. Methods: A retrospective analysis of 108 patients with IVC filters placed between 01/31/16 and 01/31/2017 at Allegheny General Hospital 
with the aim to identify: rate of inappropriate IVC filter placement, retrieval rate, barriers to retrieval, and rate of complications. Results: Indications for 22 of 108 IVC filters were compliant with current American College of Chest Physicians (ACCP) guidelines for IVC filter placement and 12 were placed for primary prophylaxis without evidence of VTE. Retrieval rate was $15.7 \%$ with average retrieval time of 7 months. Twenty two patients were anticoagulated at the time of IVC filter placement and 11 were started within 4 weeks without removal of the IVC filter. Conclusion: Most IVC filters placed at our institution are not compliant with current guidelines. There is a need to implement system-wide evidence-based policies for proper utilization of IVC filters. This will prevent potential complications, risks associated with inappropriate use of these devices, and reduce massive health care costs.

\section{3}

\section{WHEN NOTHING FITS, THINK CANCER}

Talal Khan, Saleha Rizwan and Alison O’Donnell

Allegheny Health Network, Pittsburgh, PA, U.S.A.

Background: Lambert-Eaton myasthenic syndrome (LEMS) is a rare paraneoplastic disorder in which antibodies are formed against the presynaptic voltage-gated calcium channels (VGCC). It commonly presents with proximal muscle weakness and is often associated with an underlying malignancy, like small cell lung cancer (SCLC). While muscle weakness has a broad differential diagnosis, one must keep LEMS associated with occult malignancy in the differential. Clinical predictive tools, like the DELTA-P score, can help stratify patients diagnosed with LEMS into high-risk category and trigger investigation for underlying malignancy, especially in the elderly population. Case Report: An 82-year-old African-American female with history of spinal radicular disease presented with a six-month history of progressive proximal muscle weakness. This was originally attributed to worsening radicular disease but an unrevealing magnetic resonance imaging of spine led to further investigations. A rheumatologic work-up was done, which was inconclusive except nonspecific elevated antinuclear antibodies (ANA) titers. Additionally, neurophysiological studies revealed diminished action potential in all extremities, suggestive of polyneuropathy. With unclear etiology of weakness, there was suspicion for paraneoplastic phenomenon specifically due to older age and early progression of bulbar symptoms. Thus, a computed tomography (CT) chest was performed, revealing a large central lung mass suspicious for SCLC along with strongly positive voltage gated calcium channel antibodies, indicative of LEMS. Due to the poor functional status and the preference of the patient, diagnostic biopsy was deferred and palliative radiation was planned. Discussion: Approximately, $60 \%$ of LEMS patients have underlying SCLC. Once a diagnosis of LEMS is established, delay in identification of an underlying malignancy can lead to poor patient outcomes. Neurological symptoms of LEMS usually precede anywhere from 6 months to 4-5 years before discovery of underlying malignancy. Certain factors have been proven to be strong predictors for the presence of SCLC in LEMS patients. These include current or former smoking history, age $>50$ years, weight loss, poor performance status, male impotence, raised erythrocyte sedimentation rate (ESR) and early onset of bulbar symptoms. This has culminated in the development of the Dutch-English LEMS Tumor Association Prediction (DELTA-P) score, which has a $>94 \%$ reliability of predicting SCLC in LEMS patients earlier in the course of the disease. It prioritizes high-risk patients to go through screening for underlying neoplasm via CT chest and fluorodeoxyglucosepositron emission tomography (FDG-PET) scans. A score of 0-1 reliably identifies low-risk patients who can thus be excluded. Conclusion: Muscle weakness is a common symptom with a broad differential, which can often be misleading in patients with prior neurological diagnoses. Age and risk-appropriate cancer screening should therefore be an essential part of the diagnostic work-up. This case highlights the importance of keeping a high index of suspicion for paraneoplastic phenomenon associated with underlying malignancies in patients presenting with proximal muscle weakness.

\section{4}

\section{HEPARIN RESISTANCE AND THE CHALLENGE IN MDS ASSOCIATED THROMBOEMBOLISM: COMPLICATIONS OF A COMPLICATION}

Anusha Yanamadala, Anmol Baranwal and Christine Junia

MacNeal Hospital, Berwyn, IL, U.S.A.

Background: The myelodysplastic syndrome (MDS) is a bone marrow disorder of peripheral blood cytopenias, dysplastic cells and occasionally thrombocythemia that can result in venous thrombosis. Qualitative changes in platelet function have also been proposed. Heparin resistance requiring $>35,000 \mathrm{U} / 24 \mathrm{~h}$ to achieve therapeutic activated thromboplastin time (aPTT) is best described in inherited or acquired anti-thrombin (AT) deficiency, though it is an uncommon finding in MDS. We present a case of venous thromboembolism (VTE) from MDS complicated by heparin resistance. Case Report: A 51-year-old male presented with syncope, pleuritic chest pain, and dyspnea for 2 weeks. Physical exam revealed pallor and a flow murmur. Initial studies revealed hemoglobin of $8.2 \mathrm{~g} / \mathrm{dl}$ and thrombocythemia of $575 \mathrm{~K} / \mu \mathrm{l}$. Computed tomography (CT) chest revealed pulmonary embolism and lower extremity 
ultrasound revealed bilateral acute deep vein thrombosis (DVT). Patient did not attain therapeutic aPTT $(\sim 39,000$ $\mathrm{U} / 24 \mathrm{~h}$ ) on heparin therapy and was started on argatroban treatment. Evaluation for malignancy was unremarkable. Hypercoagulability work-up was negative for factor $\mathrm{V}$ Leiden mutation, homocysteine level, and anti-cardiolipin antibodies. Patient had elevated ferritin, normal vitamin B12 and folate levels. Peripheral blood smear revealed severe thrombocythemia. Patient was discharged on warfarin. Further work-up at a tertiary care center revealed MDS. Results: Limited literature describes an increase in platelet factor 4 that neutralizes heparin in MDS, and an elevation in acute phase reactants, which decrease heparin efficacy. Given that AT deficiency usually presents with VTE at an earlier age and our work-up was negative for an identifiable cause for an acquired deficiency; heparin-resistance in this case was a possible complication of the MDS. Conclusion: This case highlights the risk of suboptimal treatment response to heparin in patients with MDS, a population with increased risk for thrombotic complications. A better understanding of the complex mechanisms of heparin resistance, particularly intensified by MDS, would help guide therapeutic management.

\section{5}

\section{MULTIPLE THYROIDAL METASTASES IN RENAL CELL CARCINOMA, A RARE OCCURANCE IN CLINICAL SETTINGS: A CASE REPORT}

Ahsan Wahab, Siddique Chaudhary, Mahin Khan, Hazif Khan, Kavitha Kesari, Susan Smith and Arvind Kunadi

McLaren Regional Medical Center, Flint, MI, U.S.A.

Background: Although reported in the literature, thyroid metastasis (TM) in renal cell carcinoma (RCC) is uncommon. We present a case of RCC with isolated TM. Methods: A 56-year-old female with abdominal pain was found to have a $2.7 \mathrm{~cm}$ right kidney mass and underwent radical nephrectomy. The tumor was staged as T1NOM0 and histopathology showed clear RCC with Fuhrman grade 2. Six years later, the patient developed respiratory distress and was diagnosed with obstructing multinodular goiter. Thyroidectomy was performed and all nodules showed metastatic disease consistent with her primary RCC. Other metastatic work-up was negative. Patient was discharged stable. Results: Primary organs that clinically manifest TM are kidney, lungs, breast, esophagus, skin, and uterus, while isolated TM can be the initial presentation of the primary. According to a previous article, only $1-4 \%$ of all metastatic RCC represent solitary sites of metastasis and only $1 \%$ of those are TM. Therefore, metastatic RCC isolated to the thyroid is very rare. TM may occur prior to, simultaneously with, or many years after the initial diagnosis of RCC.
Presentations include dysphagia, dyspnea, pain and hoarseness, though thyroid enlargement can also be the initial complaint. Ultrasound and CT are helpful in localizing lesions; fine-needle aspiration cytology (FNAC) along with immune-histochemical staining against CD-10 and vimentin differentiate RCC from thyroid tissue, which stains for thyroglobulin and thyroid transcription factor-1 (TTF-1). Treatment depends on the extent of metastases and includes surgery, adjuvant therapy, and palliative care. Solitary resectable lesions have favorable prognosis but disseminated disease is associated with a grave prognosis. Conclusion: $\mathrm{TM}$, though highly uncommon in routine clinical practice, can create confusion and diagnostic dilemma and hence, it should be considered as one of the differential diagnoses even whern there is a remote history of RCC.

16

\section{DISPARITIES IN RECEIPT OF RADIOTHERAPY AND SURVIVAL AMONG PATIENTS WITH GLIOBLASTOMA}

\author{
Niranjan Ojha ${ }^{1}$, Anu Aryal ${ }^{1}$ and Binay Kumar Shah ${ }^{2}$ \\ ${ }^{1}$ University of Washington, Seattle, WA, U.S.A.; \\ ${ }^{2}$ PeaceHealth United General Hospital, Sedro-Woolley, \\ WA, U.S.A.
}

Background: Radiotherapy is a standard therapy for glioblastoma (GBM). In this population-based study, we evaluated disparities in receipt of radiotherapy and its effect on survival among patients with GBM. Methods: Selected patients were diagnosed with GBM between January 2000 and December 2013 from the Surveillance, Epidemiology, and End Results (SEER) 18 databases. Univariate and multivariate logistic regressions were used to analyze receipt of radiotherapy by age ( $<60$ years and $\geq 60$ years), sex (male and female), race (Caucasian, African-American and other), household income $(<25,000,25,000-50,000$ and $\geq 50,000$ USD), and residence (metro and non-metro counties). SEER*stat software 8.3.2 was used to calculate 1 - and 5- year relative survival (RS) using. Cox proportional hazard model was used to determine the impact of age, sex, race, household income and residence on survival rates. Results: A total of 36,741 histologically-confirmed cases of GBM were included in the study. Among them, 25,296 $(68.8 \%)$ received radiotherapy (RT). After covariates were adjusted, we found that older patients ( $\geq 60$ years) (adjusted $\mathrm{OR}=0.430, \quad p<0.001$ ), females, (adjusted $\mathrm{OR}=0.842$, $p<0.001$ ), non-metro county residents (adjusted $\mathrm{OR}=0.899$, $p<0.05$ ) and African-American (adjusted $\mathrm{OR}=0.903$, $p<0.05)$ were less likely to receive RT. Those receiving RT were less likely to die (adjusted HR $=0.5214, p<0.001$ ). RS rates were significantly higher for patients receiving RT compared to those who did not $(42.8 \pm 3.1 \%$ vs $10.2 \pm 2.9 \%$, 
$p<0.001$ at 1 -year and $3 \pm 1.1 \%$ vs. $1.2 \pm 1.1 \%, p<0.001$ at 5-years). Conclusion: The majority (68.8\%) of patients with GBM received RT. Several factors such as age, sex, race and residence were associated with the receipt of RT. Survival rates were significantly higher for patients receiving $\mathrm{RT}$.

\section{7 \\ PERITONEAL ADENOCARCINOMA OF MULLERIAN TYPE: A RARE CASE OF BILIARY OBSTRUCTION}

Anmol Baranwal, John Berry and Ann Maria Canelas

MacNeal Hospital, Berwyn, IL, U.S.A.

Background: Peritoneal adenocarcinomas of Mullerian type (PAMT) are rare tumors similar to ovarian serous carcinomas with respect to histological presentation, treatment and prognosis. Here we present a case of PAMT with a rare biliary metastasis. Methods: A 66-year-old female presented with chief complaints of generalized abdominal pain, vomiting, diarrhea, and jaundice for past 3 weeks. Patient denied weight loss, decreased appetite or bloody stool. Physical examination was unremarkable. Patient had alkaline phosphatase of $248 \mathrm{U}$, alanine transaminase (ALT) of $160 \mathrm{U}$, aspartate transaminase (AST) of $100 \mathrm{U}$ and total bilirubin of $8.5 \mathrm{mg} / \mathrm{dl}$, while lipase was normal. Computed tomography (CT) scan of the abdomen demonstrated extensive soft tissue nodularity of anterior peritoneum and intrahepatic biliary ductal dilatation. Magnetic resonance cholangiopancreatography (MRCP) demonstrated biliary duct dilatation with abrupt transition in the region of porta hepatis suggestive of Klatskin's tumor. Measurement for cancer antigen (CA) 125 was $1613 \mathrm{U} / \mathrm{ml}$, while carcinoembryonic antigen (CEA), CA 19-9, and AFP were within normal limits. Immunohistochemical staining of the omentum supported Mullerian origin, and a final diagnosis of PAMT was established. It was uncertain whether the biliary tumor was primary or a metastasis from the peritoneal carcinoma. Biliary stents were placed and the patient was started on chemotherapy with carboplatin and paclitaxel for the peritoneal carcinoma and the response was excellent. Results: PAMT has a reported incidence of 6.8 per million. Our case posed the diagnostic dilemma of PAMT with a rare biliary metastasis, or a concurrent PAMT with Klatskin's tumor, which is rarer. In the latter case, the patient would have chemotherapy for the biliary tumor as well, which would cause additional adverse effects. The overall improvement in symptoms and functional status of the patient would not be expected if there was an additional Klatskin's tumor. Conclusion: The histology coupled with the clinical picture and therapeutic response allow us to conclude that our patient had PAMT with a rare biliary metastasis.
18

\section{SOLITARY EXTRAMEDULLARY PLASMACYTOMA OF THE THYROID GLAND REFACTORY TO RADIATION THERAPY TREATED WITH ADJUVANT BORTEZOMIB AND DEXAMETHASONE}

Subash Ghimire, Swapna Talluri, Amir Bista, Zachary Wolfe, Manidhar Lekkala, Ashit Sarker and Bradley Lash

Gurthrie Robert Packer Hospital, Sayre, PA, U.S.A.

Background: Extramedullary plasmacytomas (EMP) are rare plasma cell neoplasms (1). Involvement of the thyroid gland is extremely rare (2). We describe the case of a patient with EMP of thyroid gland that was refractory to radiation therapy, however responded to adjuvant chemotherapy with bortezomib and dexamethasone. Case Report: A 53-year-old female initially presented with complaints of difficulty swallowing, hoarseness, and neck pain for one month. Examination of neck revealed a firm and enlarged thyroid without any cervical adenopathy. A computed tomography (CT) scan of the neck was done which revealed an enlarged thyroid gland with a focal area of increased echogenicity in the left lobe. A left subtotal thyroidectomy was performed due to compression symptoms. Microscopy revealed replacement of thyroid parenchyma with sheets of mature-appearing plasma cells and few scattered thyroid follicles (Figure 1). Immunohistochemistry (IHC) confirmed plasma cells with expression of CD138 (Figure 2). Bone marrow biopsy showed a $0.2 \%$ population of polytypic plasma cells, confirmed by CD138 IHC (Figure 3). Serum protein electrophoresis, total protein, albumin, $\alpha 1, \alpha 2$ and $\beta$ globulin, calcium, and $\beta 2$-microglobulin were in normal range. Positron emission tomography (PET) scan showed no other sites of disease except the thyroid. No lytic bone lesions were present. The patient was treated with $50.4 \mathrm{~Gy}$ of radiation. Despite the surgery and radiation, the pain around the neck continued and repeat PET scan 3 months later showed persistent uptake in the thyroid. Due to refractoriness to radiotherapy, the patient was started on systemic therapy with weekly regimen of bortezomib and dexamethasone for 9 cycles. A repeat PET scan 3 months later showed no evidence of uptake suggesting adequate response to chemotherapy. Discussion: EMP encompasses $3-5 \%$ of all plasma cell neoplasms (1). The diagnostic requirements of EMP include: a) histology showing monoclonal plasma cell infiltration, b) normal plasma cell aspirate and biopsy (plasma cells $<5 \%$ ), c) no lytic lesions on skeletal survey, d) no anemia, hypercalcemia or renal impairment, and e) absent or low serum M protein (2). 


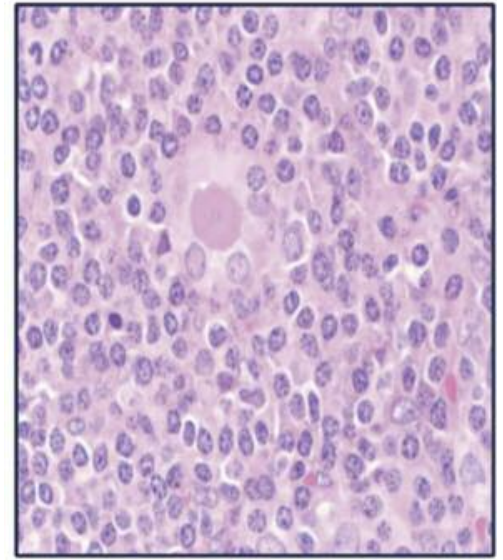

Figure 1. Mature plasma cells surrounding follicles (hematoxylin and eosin staining).

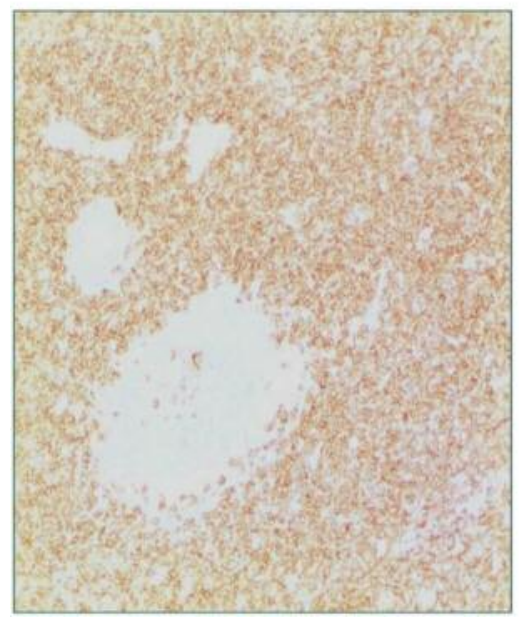

Figure 2. CD138-positive plasma cells in thyroid were confirmed by immunohistochemistry. Unstained areas show lymphoid follicles.

There exists limited evidence and experience regarding the use of chemotherapy in treatment of EMP. Some authors have reported the use of chemotherapy in plasmacytomas of pancreas and stomach (3). There is no documented literature involving treatment of EMP of thyroid with chemotherapy. In our case, despite the treatment with surgery and radiation, there was persistent uptake on PET scan. Thus, we treated with bortezomib and dexamethasone. Conclusion: EMP of the thyroid gland is a rare condition. The present case adds to the available literature, as to our knowledge, this is the only case report of using myeloma-based therapy in cases of EMP of the thyroid.

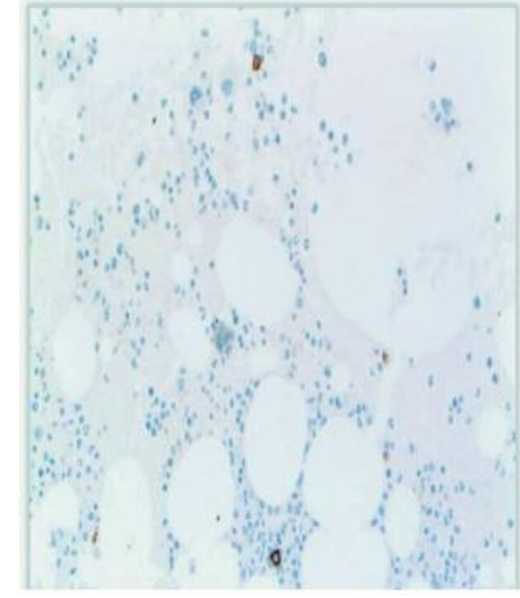

Figure 3. Immunohistochemistry showed rare CD138-positive plasma cells in bone marrow.

1 Weber DM: Solitary bone and extramedullary plasmacytoma. Hematology Am Soc Hematol Educ Program 2005: 373-376, 2005.

2 Galieni P, Cavo M, Pulsoni A, Avvisati G, Bigazzi C, Neri S, Caliceti U, Benni M, Ronconi S and Lauria F: Clinical outcome of extramedullary plasmacytoma. Haematologica 85(1): 47-51, 2000.

3 Wei JY, Tong HY, Zhu WF, Liu H, Zhang FJ, Yu WJ and Jin J: Bortezomib in treatment of extramedullary plasmacytoma of the pancreas. Hepatobiliary Pancreat Dis Int 8(3): 329-331, 2009.

19

IMMUNOPHENOTYPIC COMPARISON BETWEEN REACTIVE BONE MARROW B-LYMPHOCYTE PRECURSOR (HEMATOGONES) AND B-NEOPLASTIC LYMPHOBLAST LEUKAEMIA BY FLOW CYTOMETRY USING CD34 AND CD123 MARKERS

Raad J. Al-Ani ${ }^{1}$, Yousra A. Shallan ${ }^{2}$ and Mohammed R. Al-Ani ${ }^{3}$

${ }^{1}$ Isra University - College of Pharmacy, Amman, Jordan; ${ }^{2}$ Najaf Health Office, Al-Najaf, Iraq;

${ }^{3} \mathrm{Al}-$ Karak Governmental Hospital, Al-Karak, Jordan

Background: Flow cytometric study has found that lymphoblasts of B-acute lymphoblastic leukemia expressed multiple aberrant antigens by which they can be distinguished from hematogones. These antigens are CD34 and CD123. Aim: To determine the pattern of CD34 and 
CD123 expression in hematogones of reactive bone marrow and in neoplastic lymphoblasts in B-acute lymphoblastic leukemia (B-ALL) patients, and evaluate the impact of that pattern in the residual disease detection after chemotherapy. Methods: The expression of CD34 and CD123 was determined in 30 patients newly diagnosed with B-ALL. Reassessment was done for 20 patients of them after 4-6 weeks of chemotherapy; in addition to 10 patients with reactive bone marrow to assess hematogones. Results: In the $93.4 \%$ of the newly diagnosed B-ALL cases, leukemic blasts expressed both CD34 and CD123. Conversely, in $6.6 \%$ of cases, neither antigen was expressed. In hematogones, the immature hematogones (dim CD45, CD34+) did not express CD123, while the mature hematogones (moderate $\mathrm{CD} 45^{+}, \mathrm{CD} 34^{-}$) expressed CD123. The strategy of concordant and discordant patterns of CD34/CD123 expression on B-ALL blasts and hematogones respectively in post-chemotherapy patients remain stable. Conclusion: The distinct pattern of CD34 and CD123 expression on hematogones (discordant) and B-ALL blasts (concordant) is useful in correctly classifying immature $\mathrm{B}$ cells as residual leukemic blasts or hematogones in the bone marrow of patients treated for B-ALL.

\section{0}

\section{A RARE CASE OF PRIMARY SUB-MUCOSAL RECTAL SQUAMOUS CELL CARCINOMA DIAGNOSED WITH ENDOSCOPIC ULTRASOUND}

Prince Sethi ${ }^{1}$, Khalil Aloreidi ${ }^{1}$, Radowan Elnair ${ }^{1}$, Usama Yassi ${ }^{2}$, Muslim Atiq ${ }^{3}$ and Bhavesh Patel ${ }^{3}$

${ }^{1}$ Internal Medicine Residency Program, University of South Dakota Sanford School of Medicine,

Sioux Falls, SD, U.S.A.;

${ }^{2}$ Pathology Residency Program, University of South

Dakota Sanford School of Medicine,

Sioux Falls, SD, U.S.A.;

${ }^{3}$ Sanford Center for Digestive Health,

Sioux Falls, SD, U.S.A.

Background: A squamous cell carcinoma (SCC) of the rectum is a rare primary colorectal malignancy. Here we report a case of primary submucosal SCC of the rectum treated with chemo-radiation. Case Report/Results: A 56year-old female with no significant past medical history presented with complaints of generalized abdominal discomfort with onset few weeks ago. Computed tomography (CT) of abdomen and pelvis with intravenous contrast showed soft tissue mass measuring around $20 \mathrm{~mm}$ at the left lateral wall of the rectum. Patient underwent colonoscopy and rectal ultrasound showing two hypoechoic lesions (largest of size 16-17 $\mathrm{mm}$ and smaller lesion around $10 \mathrm{~mm}$ ) at around $10 \mathrm{~cm}$ from the anal verge. Both lesions were in close proximity and appeared to be originating from the muscularis propria layer. Fine needle aspiration was performed separately from both lesions with pathology consistent with squamous cell carcinoma. No significant perirectal or iliac lymphadenopathy was noticed on rectal ultrasound. Positron emission tomography (PET)/CT scan showed hyper-metabolic rectal lesion consistent with known malignancy with additional foci of hyper-metabolism in the perirectal fat concerning for nodal disease without distant metastases (T2 N1 M0). The patient underwent chemotherapy (mitomycin-C with 5-fluorouracil) and radiation therapy. Surveillance PET-CT showed no residual lesions in rectal wall. Conclusion: Primary rectal SCC is a distinct and extremely rare form of colorectal malignancy making the presentation of sub-mucosal rectal SCC an even rarer entity. Etiopathogenesis of rectal SCC is poorly understood. An important step in diagnosis is the exclusion of other primary SCC with metastasis to gastrointestinal tract. Endoscopic ultrasound and PET-CT provide important diagnostic information to plan for further management. There has been a shift in preference towards definitive chemo-radiation for rectal SCC, simulating anal canal SCC standard of care. Due to the paucity of literature the treatment plan at this time should be individualized based on the presentation of the patient.

\section{1}

\section{REGULATION OF ID-1 GENE EXPRESSION IN AGGRESSIVE BREAST CANCER AND GLIOBLASTOMA CELLS USING CANNABINOID COMPOUNDS}

\section{Ogechukwu Kingsley Egini}

Interfaith Medical Center, Brooklyn, NY, U.S.A.

Background: The inhibitor of DNA binding-1 (ID-1) encodes a helix-loop-helix (HLH) protein that has no DNA binding ability, thus inhibiting DNA binding and transcriptional activity of the HLH proteins with which it interacts. Downregulating the expression of $I D-1$ using antisense technology significantly impaired breast cancer cell proliferation and invasion in culture. Cannabinoids were discovered as potential candidates to down-regulate ID-1 expression in metastatic cancers. Several of these compounds are agonists of two $G$ protein-coupled receptors, $\mathrm{CB} 1$ and $\mathrm{CB} 2$. These receptors have tumorinhibiting ability. In this study, cannabidiol (CBD) and tetrahydrocannabinol (THC) were used as cannabinoid agents, while ethanol served as control. The aim was to investigate ID1 expression in aggressive breast cancer (MDA-MB231) and glioblastoma (U251) cell lines and to compare ID-1 expression in control versus CBD and THC-treated cells. Methods: The tumor cells were treated with CBD or THC, and the control group was treated with ethanol. After 2-3 days of treatment, morphological changes of treated and control cells were 
A
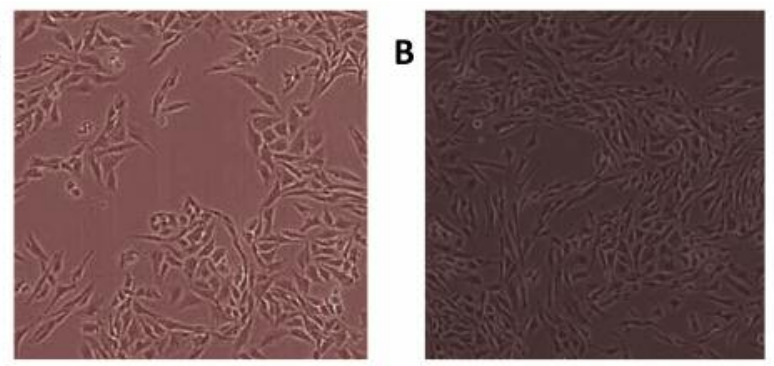

Figure 1. Ethanol-treated U251 cells taken on the 2nd (A) and 3rd (B) day of treatment. Control cells have spindle morphology.

A

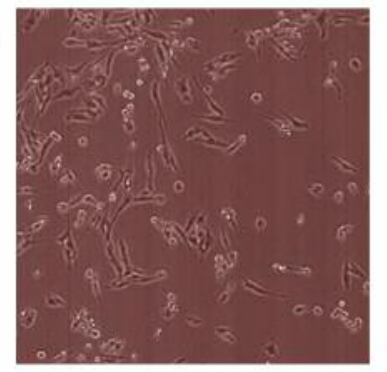

B

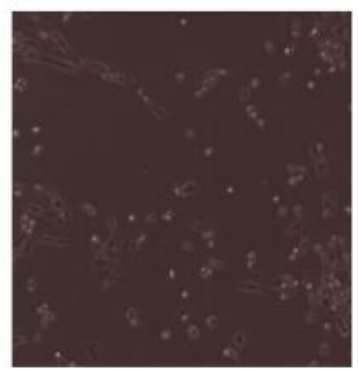

Figure 2. CBD-treated U251 cells on the 2nd (A) and 3rd (B) day of treatment. Morphology of cells is oval.
A

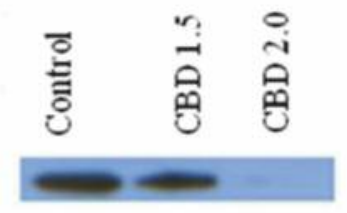

B

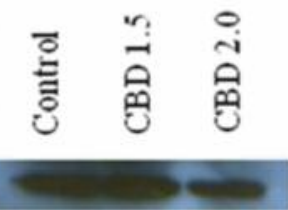

Figure 3. ID-1 blots of U251 cells following a 2-day treatment with ethanol or CBD. Panel A shows the ID-1 blot and B shows the actin blot.

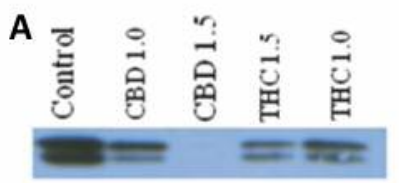

B

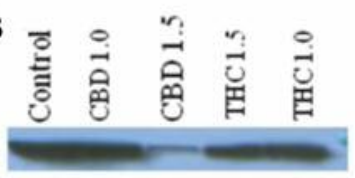

Figure 4. ID-1 blots of MDA-MB231 cells following a 2-day treatment with ethanol, CBD or THC. Panel A shows the ID-1 blot and $B$ shows the actin blot. examined, followed by protein electrophoresis and western blotting to analyze the expression of ID-1 protein derived from the samples. Band intensities produced following film exposure were used to compare cannabinoid drug-treated cells with controls. Results: ID-1 expression was decreased in tumor cells treated with cannabinoid compounds, more in CBD-treated than THC-treated tumor cells. In addition, cell proliferation was remarkably reduced in glioblastoma cells treated with CBD (Figures 1 and 2). The cell morphology of the glioblastoma cells treated with CBD also changed from the normal spindle-shaped morphology to a more oval morphology (Figures 3 and 4). Conclusion: ID-1 downregulation strongly correlates with reduced tumor aggressiveness; hence ID-1 might represent a potent therapeutic target for breast cancer and glioblastoma.

\section{2}

\section{DIFFUSE B-CELL LYMPHOMA OF SPHENOID SINUS: AN UNUSUAL PRESENTATION OF B CELL LYMPHOMA}

Sara Elsayed, Raathathulaksi Krishnamohan and Prabhat Pokhrel

Mclaren Flint - Family Medicine Residency Program, Flint, MI, U.S.A.

Background: Diffuse large B-cell lymphoma (DLBCL) is the most common lymphoma and accounts for approximately $25 \%$ of all non-Hodgkin's lymphomas (NHLs) in the developed world. In the U.S., the incidence of DLBCL is approximately 7 cases per 100,000 persons per year. Extranodal extramedullary disease occurs in up to $40 \%$ of cases. The most common site of extranodal involvement is the gastrointestinal tract, but the disease can arise in virtually any tissue. DLBCL is a heterogeneous group of tumors consisting of large, transformed B-cells with prominent nucleoli and basophilic cytoplasm, a diffuse growth pattern and a high proliferation fraction. DLBCL arises from a mature B-cell, and is usually comprised of cells resembling centroblasts or immunoblasts. Case Report: A 74-year-old Caucasian male with a background of Huntington's disease presented to the clinic with intermittent loss of vision of the left eye for 3 weeks. The patient was evaluated by his ophthalmologist and was advised to followup with primary care physician. The patient presented to the hospital due to worsening intermittent vision loss and headache. There was no history of fever, weight loss, or nocturnal sweating. On physical examination, the patient had generalized weakness and slurred speech secondary to Huntington's disease. Initial computed tomography (CT) scans of the head showed severe bilateral sinusitis and no acute intracranial process. Neurologist recommended aspirin and patient was discharged home after resolution of his vision loss with the diagnosis of amaurosis fugax. Patient was readmitted 
after two months for generalized weakness. CT of the head, that was done to rule-out a stroke, showed marked opacification of the sphenoid sinus with soft tissue density extending into the left orbital apex. MRI confirmed a mass in the left sphenoid sinus extending to the left orbit with a mass effect on the left medial rectus and left optic nerve. Results: Sinus endoscopy and biopsy of the mass extending to the orbit confirmed DLBCL germinal center type with marker of proliferation $\mathrm{Ki}-67$ of $10 \%$. Immunostains were positive for CD20, CD79a, CD10, BCL-6 and BCL-2. Hematology/oncology was consulted, and radiation therapy in combination with chemotherapy was recommended. Rituximab and bendamustine was preferred over rituximab plus cyclophosphamide/adriamycin/vincristine/prednisolone (RCHOP) because of the underlying Huntington's disease. Conclusion: The incidence of non-Hodgkin lymphoma is increasing more rapidly than many other malignancies. The estimated rate of DLBCL is approximately 4.68 cases per 100,000 person years. Although, DLBCL is the most common lymphoma of all NHLs in the developed world and it can potentially involve any site of the body, involvement of the orbit is not a common presentation. To our knowledge, only a limited number of cases with DLBCL of sphenoid sinus have been found. The proximity of the optical nerve to the sphenoid sinus is responsible for the high incidence of visual disturbance in patients with lymphomas at this site. Radiological imaging has a major role in initial diagnosis of non-Hodgkin's lymphoma. It can also provide information on the extension of the tumor and involvement of bony structure. Biopsy for histopathological evaluation and immunostaining is essential in diagnosing non-Hodgkin lymphoma. It was found that DLBCL with germinal center phenotype has better outcomes compared to non-germinal center phenotype. Ki-67 is a nuclear protein involved in cell proliferation regulation, and its expression has been widely used as an index to evaluate the proliferative activity of lymphoma. The prognostic significance of Ki-67 expression in DLBCL is controversial. NonHodgkin's lymphoma is frequently treated with a combination of chemotherapy and radiotherapy. Despite advancements in the treatment of DLBCL, approximately $40 \%$ of patients relapse after treatment or are refractory to treatment. Research suggests that treatment with $\mathrm{CHOP}$ regimen results in the best outcome. When immunostaining is positive for CD20, rituximab, which is directed against CD20 surface antigen, in addition to $\mathrm{CHOP}$ regimen increase the chances of a better outcome. This is a unique case because of the rare involvement of the sphenoid sinus and the non-specific presenting symptoms. Due to the rare occurrence of DLBCL in sphenoid sinus, the presentation can be easily misinterpreted as transient ischemic attack. In patients with confounding comorbidities that symptoms could be attributed to, other rare causes such as DLBCL of the sinus must be considered and ruled out even in the absence of systemic findings.
1 Bisdas S, Fetscher S, Feller AC, Baghi M, Knecht R, Gstoettner W, Vogl TJ and Balzer JO: Primary B cell lymphoma of the sphenoid sinus: CT and MRI characteristics with correlation to perfusion and spectroscopic imaging features. Eur Arch Otorhinolaryngol 264: 1207-1213, 2007.

2 Coiffier B, Lepage E and Briere J: CHOP Chemotherapy plus rituximab compared with CHOP alone in elderly patients with diffuse large B-cell lymphoma. N Engl J Med 346: 235-242, 2002.

3 Freeman C, Berg JW and Cutler SJ: Occurrence and prognosis of extranodal lymphomas. Cancer 29: 252-260, 1972.

4 Frierson HF Jr., Mills SE, Innes DJ: Non-Hodgkin's lymphomas of the sinonasal region: Histologic subtypes and their clinicopathologic features. Am J Clin Pathol 81: 721-727, 1984.

5 Jacobs C and Hoppe RT: Non-Hodgkin's lymphomas of head and neck extranodal sites. Int J Radiat Oncol Biol Phys 11: 357-364, 1985.

6 Morton LM, Wang SS, Devesa SS, Hartge P, Weisendurger DD and Linet MS: Lymphoma incidence patterns by WHO subtype in the United States, 1992-2001. Blood 107: 265276, 2006.

7 Park YM, Cho JH, Cho JY, Huh JS and Ahn JY: NonHodgkins lymphoma of the sphenoid sinus presenting as isolated oculomotor nerve palsy. World J Surg Oncol 5: 86, 2007.

8 Roth DB and Siatkowski RM: Bilateral blindness as the initial presentation of lymphoma of the sphenoid sinus. Am J Ophthalmol 129: 256-258, 2000.

9 Salles G, de Jong D, Xie W, Rosenwald A, Chhanabhai M, Gaulard P, Klapper W, Calaminici M, Sander B, Thorns C, Campo E, Molina T, Lee A, Pfreundschuh M, Horning S, Lister A, Sehn LH, Raemaekers J, Hagenbeek A, Gascoyne RD and Weller E: Prognostic significance of immunohistochemical biomarkers in diffuse large B-cell lymphoma: a study from the Lunenburg Lymphoma Biomarker Consortium. Blood 117: 7070-7078, 2011.

\section{3 \\ SPONTANEOUS TUMOR LYSIS SYNDROME IN METASTATIC BREAST CANCER}

Millicent Amankwah, Jite T. Wanogho, Adewale Odofin and Radhika Khwaja

Department of Medicine, St. Vincent's Medical Center, Bridgeport, CT, U.S.A.

Background: Tumor lysis syndrome (TLS) is an oncological emergency, first described in the 1900's, even before the use of chemotherapy in the treatment of solid and other hematological tumors. It is characterized by hyperkalemia, 
hyperuricemia, hyperphosphatemia, and hypocalcemia. Case Report: A 70-year-old female with history of recurrent triplenegative, invasive ductal carcinoma of the left breast, status post mastectomy, chemotherapy, hormonal therapy, and bony metastatic disease, presented with progressive dyspnea, fatigue, and headaches. On exam, the patient looked ill, moderately dehydrated with absent breath sounds on the left hemithorax. Laboratory evaluation showed leukocytosis with neutrophilia, anemia, thrombocytosis, hyperkalemia, elevated blood urea nitrogen (BUN) and creatinine, hypocalcemia, hyperphosphatemia and hyperuricemia. Thev patient had no arrhythmia or seizure activity. Further work-up showed large hemorrhagic pleural effusion and, metastases to the dura. The patient was diagnosed with spontaneous TLS in the setting of metastatic breast cancer disease and was treated with intravenous fluids and allopurinol. The patient received palliative radiotherapy and chemotherapy without recurrence of TLS. Discussion: TLS refers to a condition in which cancer cells, spontaneously or after therapy, release their cellular contents into blood stream leading to various biochemical disturbances. Cairo and Bishop's classification using either clinical or laboratory parameters are used to define TLS. It has typically been associated with bulky hematological malignancies such as Burkitt lymphoma and leukemias; however notable literature reports of TLS in solid organ tumors exist. In reported cases of breast cancerassociated TLS, the incidence of TLS cannot be defined. There are few case reports in the literature, in which mean age of the patients is 54 years-old and all the cases have been in metastatic adenocarcinoma involving the breast. In the reported instances, the majority of TLS occurred after chemotherapy or hormonal therapy had been initiated. In our case, TLS occurred prior to initiation of chemotherapy or radiotherapy. The large tumor burden indicated by the extent of metastatic disease, elevated lactate dehydrogenase, predisposed our patient to TLS. The patient responded to treatment after prompt initiation of intravenous fluids and allopurinol. Conclusion: Early suspicion and diagnosis of the TLS in solid organ tumors is key to prevention and management. 